Research Article

\title{
The Reverse of the Intermediate Value Theorem in Some Topological Spaces
}

\author{
Oussama Kabbouch (iD) and Mustapha Najmeddine
}

My Ismail University, Ensam-Meknès Marjane, Meknès, Morocco

Correspondence should be addressed to Oussama Kabbouch; oussama.kabbouch@gmail.com

Received 4 May 2020; Revised 7 March 2021; Accepted 23 March 2021; Published 12 April 2021

Academic Editor: Frédéric Mynard

Copyright (c) 2021 Oussama Kabbouch and Mustapha Najmeddine. This is an open access article distributed under the Creative Commons Attribution License, which permits unrestricted use, distribution, and reproduction in any medium, provided the original work is properly cited.

Any continuous function with values in a Hausdorff topological space has a closed graph and satisfies the property of intermediate value. However, the reverse implications are false, in general. In this article, we treat additional conditions on the function, and its graph for the reverse to be true.

\section{Introduction}

The motivation of this work derives from the intermediate value theorem (IVT) and the closed graph theorem [1]. We discuss the results presented in [2], showing that the reverse of the IVT may be true under certain additional assumptions.

A continuous function in a Hausdorff space is known to satisfy the following facts:

(1) The intermediate value property (IVP).

(2) Its graph is closed.

(3) The inverse image of each closed set is closed.

The reverse of the two first results is false in general. That means if a function satisfies the IVP or its graph is closed, it is not necessarily continuous. The objective of this work is to provide some conditions in which this reverse is true. In this sense, we prove in Theorem 2 that every function from a locally connected metric space into a locally sequentially compact space, which has a sequentially closed graph and satisfies the IVP, is continuous. Theorem 1 is a generalization of Theorem 2 for functions having a closed graph from locally connected space into locally compact spaces. In Theorem 1, we have a generalization in normed spaces of the result given in [2]. In Theorem 6, we show that the sequential closeness of the graph implies that the inverse image of a sphere is sequentially closed.
We know that the closeness of the graph implies the continuity of linear maps between Banach spaces [3]. The same result is given in Corollary 2 for functions, not necessarily linear, between normed spaces satisfying the IVP in the case if the codomain is a finite dimensional normed space.

From now, Let $E$ and $F$ be Hausdorff topological spaces and $f$ be a map from $E$ into $F$. The graph of $f$ is defined by

$$
\Gamma_{f}=\{(x, f(x)): x \in E\} .
$$

Definition 1. Let $X$ be a Hausdorff topological space.

(1) The function $f$ is said to be satisfying the intermediate value property (IVP) if the image of every connected subset of $E$ is connected in $F$.

(2) A subset $A$ of $X$ is said to be sequentially closed if it contains the limit of every convergent sequence $\left(a_{n}\right)_{n \in \mathbb{N}}$ in $A$.

(3) A subset $A$ of $X$ is said to be sequentially compact if every sequence in $A$ admits a limit point in $A$.

(4) The space $X$ is said to be locally sequentially compact if every $x \in X$ admits a basis of sequentially compact neighbourhoods.

(5) The space $X$ is said to be locally connected if every $x \in X$ admits a basis of connected neighbourhoods. 
Remark 1. Every sequentially closed subspace of a locally sequentially compact space is locally sequentially compact.

In [1], we have the following classical results.

Proposition 1. If $f$ is continuous, then its graph is closed and it satisfies the IVP.

Theorem 1. Let $C$ be a connected subset in a topological space $X$. For every subset $A$ of $X$ such that $C \cap A \neq \varnothing$ and $C \cap(X \backslash A) \neq \varnothing$, we have $C \cap \operatorname{Fr}(A) \neq \varnothing$, where $\operatorname{Fr}(A)=\bar{A} \backslash \AA$.

Now, consider the following examples.

Example 1. (1) The function

$$
f(x)= \begin{cases}\sin \left(\frac{1}{x}\right), & \text { if } x \neq 0, \\ 0, & \text { if } x=0\end{cases}
$$

satisfies the IVP, but it is not continuous at 0 .

(2) The function

$$
g(x)= \begin{cases}\frac{1}{x}, & \text { if } x \neq 0 \\ 0, & \text { if } x=0\end{cases}
$$

has a closed graph, but it is not continuous at 0 .

(3) By the Darboux theorem [4] (asserting that the derivative of a differentiable function on the real line satisfies the IVP), the derivative of any real function which is not in $\mathscr{C}^{1}$ on the real line satisfies the IVP but it is not continuous.

(4) Using the expansion of reals in basis 13, the work given in [5] gives a construction of functions satisfying the IVP which are nowhere continuous.

(5) Let $I$ be an uncountable set and $\mathbb{K}$ be the real or the complex field. Set $E=\mathbb{K}^{(I)}=\oplus_{i \in I} \mathbb{K}_{i} \quad$ with $\mathbb{K}_{i}=\mathbb{K}, i \in I$ and

$$
F=\left\{\left(x_{i}\right)_{i \in I} \in \mathbb{K}^{I}:\left\{i \in I: x_{i} \neq 0\right\} \text { is at most countable }\right\} \text {. }
$$

Then, $E$ is a linear subspace of the space $\ell^{\infty}(I)$ of bounded sequences in $\mathbb{K}^{I}$. Define a duality between $E$ end $F$ for all $x=\left(x_{i}\right)_{i \in I} \in E$ and $y=\left(y_{i}\right)_{i \in I} \in F$ by

$$
\langle x, y\rangle=\sum_{i \in I} x_{i} y_{i}
$$

The sets

$$
W(A, \varepsilon)=\{y \in F:|\langle x, y\rangle|<\varepsilon \text { for all } x \in A\},
$$

where $A$ is a finite subset of $E$ and $\varepsilon>0$, form a basis of a zero neighbourhood of a linear topology on $F$ called the weak topology on $F$ with respect to the duality between $E$ and $F$ denoted by $\sigma(F, E)$.

The sets $W(K, \varepsilon)$ where $K$ is a convex balanced compact subset in the weak topology of $F$ and $\varepsilon>0$ form a basis of a zero neighbourhood of a linear topology on $E$ called the Mackey topology on $E$ with respect to the duality between $E$ and $F$ denoted by $\tau(E, F)$.

The Mackey topology on $E$ is the strongest topology for which the topological dual is the space of the linear maps

$$
\varphi_{y}: E \longrightarrow \mathbb{R}, x \mapsto\langle x, y\rangle, \quad y \in F
$$

Let $E$ be provided with the Mackey topology $\tau(E, F)$ and $\ell^{1}(I)$ be the Banach space of summable families $\left(x_{i}\right)_{i \in I}$. The canonical injection from $E$ into $\ell^{1}(I)$ is a map with a closed graph that is not continuous. For more details, see [6].

In the following, we study conditions making the reverse of IVT true.

Theorem 2. Suppose that $(E, d)$ is a locally connected metric space and $F$ is locally sequentially compact. If $f$ satisfies the IVP and its graph is sequentially closed, then $f$ is continuous.

Proof. Suppose that $f$ is not continuous at $a \in E$. Since $E$ is locally connected, there exists a connected neighbourhood $C_{0}$ of $a$ such that

$$
C_{0} \subset B(a, 1)=\{x \in E, d(a, x)<1\} .
$$

Set $U=C_{0} \cap B(a,(1 / 2))$; then, $U$ is neighbourhood of $a$. Therefore, there exists a connected neighbourhood $C_{1}$ of $a$ such that $C_{1} \subset U$, and so we construct a decreasing sequence $\left(C_{n}\right)_{n \in \mathbb{N}}$ of connected neighbourhoods of $a$ such that

$$
C_{n} \subset B\left(a, \frac{1}{2^{n}}\right), \quad \text { for all } n \in \mathbb{N} \text {. }
$$

On the other hand, since $f$ is not continuous at $a$ and $F$ is locally sequentially compact, there exists a sequentially compact neighbourhood $V$ of $f(a)$ such that

$$
f\left(C_{n}\right) \not \subset V, \quad \text { for all } n \in \mathbb{N} \text {. }
$$

Then,

$$
f(a) \in f\left(C_{n}\right) \cap V, f\left(C_{n}\right) \cap(F \backslash V) \neq \varnothing, \quad \text { for all } n \in \mathbb{N} .
$$
have

Since $f\left(C_{n}\right)$ is connected for all $n \in \mathbb{N}$, by Theorem 1 , we

$$
f\left(C_{n}\right) \cap \operatorname{Fr}(V) \neq \varnothing, \quad \text { for all } n \in \mathbb{N} .
$$

Hence, for all $n \in \mathbb{N}$, there exists $a_{n} \in C_{n}$ such that $f\left(a_{n}\right) \in \operatorname{Fr}(V)$. With the sequential compactness of $\operatorname{Fr}(V)$, there is a subsequence $\left(f\left(a_{h(n)}\right)\right)_{n \in \mathbb{N}}$ of $\left(f\left(a_{n}\right)\right)_{n \in \mathbb{N}}$ which converges to $b \in \operatorname{Fr}(V)$. Since the sequence 
$\left(a_{h(n)}, f\left(a_{h(n)}\right)\right)_{n \in \mathbb{N}}$ is in $\Gamma_{f}$ and converges to $(a, b)$, then $b=$ $f(a)$ and $f(a) \in \operatorname{Fr}(V)$, which is a contradiction because $V$ is a neighbourhood of $f(a)$. Then, $f$ is continuous at $a$.

Remark 2. By the previous theorem,

(1) The graph of the function $f$ given in Example 1 is not a closed set.

(2) The function $g$, given in Example 2, does not satisfy the IVP.

Theorem 3. Let $E$ be a locally connected topological space and $F$ be locally compact. If $f$ satisfies the IVP and its graph is closed, then $f$ is continuous.

Proof. Suppose that $f$ is not continuous at $a \in E$. Since $F$ is locally compact, there is a compact neighbourhood $V$ of $f(a)$ such that $f(U) \not \subset V$ for all neighbourhood $U$ of $a$. By the local connectedness of $E$, there is a generalized sequence $\left(U_{\alpha}\right)_{\alpha \in \Lambda}$ of connected neighbourhoods of $a$, which is a basis of neighbourhoods of $a$ in $E$. Then,

$$
f(a) \in f\left(U_{\alpha}\right) \cap V, f\left(U_{\alpha}\right) \cap(F \backslash V) \neq \varnothing, \quad \text { for all } \alpha \in \Lambda .
$$

Since $f$ satisfies the IVP, $f\left(U_{\alpha}\right)$ is connected for all $\alpha \in \Lambda$. By Theorem 1 , there exists a generalized sequence $\left(x_{\alpha}\right)_{\alpha \in \Lambda}$ such that

$$
x_{\alpha} \in U_{\alpha}, f\left(x_{\alpha}\right) \in \operatorname{Fr}(V), \quad \text { for all } \alpha \in \Lambda .
$$

By the compactness of $V, \operatorname{Fr}(V)$ is compact. Then, there exists a subsequence $\left(x_{\alpha}\right)_{\alpha \in \Delta \subset \Lambda}$ such that $\left(f\left(x_{\alpha}\right)\right)_{\alpha \in \Delta}$ converges to $b \in \operatorname{Fr}(V)$. Then, the generalized sequence $\left(x_{\alpha}, f\left(x_{\alpha}\right)\right)_{\alpha \in \Delta}$ converges to $(a, b)$. Since $\Gamma_{f}$ is closed, then $(a, b) \in \Gamma_{f}$. Therefore, $b=f(a)$ and $f(a) \in \operatorname{Fr}(V)$, which contradicts that $V$ is a neighbourhood of $f(a)$. Thus, $f$ is continuous.

Since the normed spaces are locally connected and the finitely dimensional normed spaces are locally compact, we have the following corollary.

Corollary 1. Each function from a normed vector space into a finitely dimensional normed vector space which satisfies the IVP and with closed graph is continuous.

In [2], the following theorem gives the reverse of the IVT under weak assumptions.

Theorem 4. Let $f$ be a real valued function on an interval I of $\mathbb{R}$ satisfying the IVP. If for all $y \in \mathbb{R}, f^{-1}(\{y\})$ is closed in $I$, then $f$ is continuous.

Proposition 2. Let $f: E \longrightarrow F$ be a function; then:

(1) If the graph of $f$ is closed, then for every $y \in F, f^{-1}(\{y\})$ is closed in E.

(2) If the graph of $f$ is sequentially closed, then for every $y \in F, f^{-1}(\{y\})$ is sequentially closed in $E$.
(3) If $f$ is continuous, then for every $y \in F, f^{-1}(\{y\})$ is closed in $E$.

Proof

(1) Let $\left(x_{\alpha}\right)_{\alpha \in \Lambda}$ be a generalized sequence in $f^{-1}(\{y\})$ that converges to $x$. $\left(x_{\alpha}, y\right)_{\alpha \in \Lambda}$ is a sequence in $\Gamma_{f}$ that converges to $(x, y)$. Since $\Gamma_{f}$ is closed, then $f(x)=y$. Hence, $x \in f^{-1}(\{y\})$. Therefore, $f^{-1}(\{y\})$ is closed in $E$.

(2) In the same way, we show (2).

(3) The continuity of $f$ implies that the graph of $f$ is closed, and then we have (3).

Remark 3

(1) The reverse of 1 and 2 is false, in general. As in Example 2, since $f$ is injective, $f^{-1}(\{y\})$ is closed in $\mathbb{R}$, but $f$ has no closed graph.

(2) Theorem 2 is a corollary of Theorem 4 and Proposition 2.

The following theorem is a generalization of the real case in Theorem 4.

Theorem 5. Suppose that $F$ is a normed vector space over $\mathbb{R}$ or $\mathbb{C}$ and $E$ is locally connected metric space. If $f$ satisfies the IVP and the inverse image of every sphere in $F$ is sequentially closed in $E$, then $f$ is continuous.

Proof. Suppose that $f$ is not continuous at $a \in E$. As in the proof of Theorem 2, there is $r>0$ and a sequence $\left(a_{n}\right)_{n \in \mathbb{N}}$ in $E$ that converges to $a$, and for all $n \in \mathbb{N}, f\left(a_{n}\right) \in \operatorname{Fr}(V)=S$, where $V=B(f(a), r)$ and $S(f(a), r)$ are the ball and the sphere of radius $f(a)$, respectively. Hence, $\left(a_{n}\right)_{n \in \mathbb{N}}$ is in $f^{-1}(S)$ which is closed. Then, $a \in f^{-1}(S)$ and $f(a) \in S$ : a contradiction. Thus, $f$ is continuous.

Theorem 6. Suppose that $F$ is a finite dimensional normed vector space. If the graph of $f$ is sequentially closed, then the inverse image of every sphere in $F$ is sequentially closed in $E$.

Proof. Let $S=S(a, r)=\{x \in F:\|x-a\|=r\}$ be the sphere of center $a$ and of radius $r>0$. Let $\left(x_{n}\right)_{n \in \mathbb{N}}$ be a sequence in $f^{-1}(S)$ that converges to $x$. Let us show that $x \in f^{-1}(S)$. We know that the sequence $\left(f\left(x_{n}\right)\right)_{n \in \mathbb{N}}$ is in $S$. Since $F$ is a finite dimensional normed space, then $S$ is compact. Hence, there is a subsequence $\left(f\left(x_{h(n)}\right)\right)_{n \in \mathbb{N}}$ of $\left(f\left(x_{n}\right)\right)_{n \in \mathbb{N}}$ that converges to $y \in S$. Since the graph $\Gamma_{f}$ of $f$ is sequentially closed and $\left(x_{h(n)}, f\left(x_{h(n)}\right)\right)_{n \in \mathbb{N}}$ is a sequence in $\Gamma_{f}$ that converges to $(x, y)$, then $(x, y)$ in $\Gamma_{f}$ and $y=f(x)$. Hence, $f(x) \in S$ and $x \in f^{-1}(S)$. Therefore, $f^{-1}(S)$ is sequentially closed in $E$.

Theorem 7. Suppose that $F$ is a finite dimensional normed space. If the graph of $f$ is closed, then the inverse image of every sphere in $F$ is closed in $E$. 
Proof. Let $S=S(a, r)=\{x \in F:\|x-a\|=r\}$ be the sphere of center $a$ and of radius $r>0$. Let $x$ be in the closure $\overline{f^{-1}(S)}$; then, there exists a generalized sequence $\left(x_{\alpha}\right)_{\alpha \in \Lambda}$ in $f^{-1}(S)$ that converges to $x$. Then, the sequence $\left(f\left(x_{\alpha}\right)\right)_{\alpha \in \Lambda}$ is in $S$ which is compact. Hence, there is a subsequence $\left(f\left(x_{\alpha}\right)\right)_{\alpha \in \Delta \subset \Lambda}$ that converges to $y \in S$. The graph $\Gamma_{f}$ of $f$ is closed and $\left(x_{\alpha}, f\left(x_{\alpha}\right)\right)_{\alpha \in \Delta}$ is in $\Gamma_{f}$; then, $y=f(x)$. Hence, $f(x) \in S$ and $x \in f^{-1}(S)$. Therefore, $f^{-1}(S)$ is closed in $E$.

Corollary 2. Suppose that $F$ is a finite dimensional normed space over $\mathbb{R}$ or $\mathbb{C}$ and $E$ is locally connected metric space. If $f$ satisfies the IVP and its graph is sequentially closed, then $f$ is continuous.

\section{Data Availability}

The data used to support the findings of this study have not been made available because they are confidential.

\section{Conflicts of Interest}

The authors declare that they have no conflicts of interest.

\section{References}

[1] N. Bourbaki, Elements of Mathematics: General Topology, Springer, Berlin, Germany, 1995.

[2] W. Rudin, Principles of Mathematical Analysis, McGraw-Hill, New York, NY, USA, 1964.

[3] L. Schwartz, Analyse: Topologie Génerale Et Analyse Fonctionnelle, Hermann, Paris, France, 1970.

[4] L. Olsen, "A new proof of darboux's Theorem," The American Mathematical Monthly, vol. 111, no. 8, pp. 713-715, 2004.

[5] G. Oman, "The converse of the intermediate value Theorem: from Conway to Cantor to cosets and beyond," Missouri Journal of Mathematical Sciences, vol. 26, no. 2, pp. 134-150, 2014.

[6] A. Marquina, "A note on the closed graph Theorem," Archiv der Mathematik, vol. 28, no. 1, 1977. 